\title{
Potential for luminosity improvement for low-energy RHIC operations with long bunches
}

\author{
A. Fedotov and M. Blaskiewicz
}

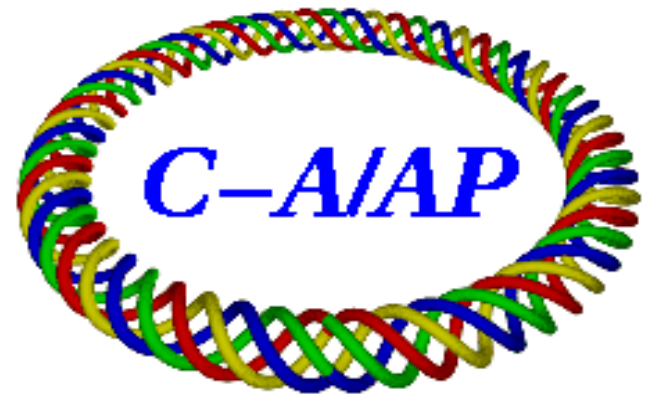

Collider-Accelerator Department Brookhaven National Laboratory

Upton, NY 11973

Notice: This document has been authorized by employees of Brookhaven Science Associates, LLC under Contract No. DE-AC02-98CH10886 with the U.S. Department of En ergy. The United States Government retains a nonexclusive, paid-up, irrevocable, world-wide license to publish or reproduce the published form of this document, or allow others to do so, for United States Government purposes. 


\title{
POTENTIAL FOR LUMINOSITY IMPROVEMENT FOR LOW-ENERGY RHIC OPERATION WITH LONG BUNCHES
}

\author{
Alexei Fedotov and Mike Blaskiewicz \\ $C$-AD, Brookhaven National Laboratory
}

\section{Introduction}

Electron cooling was proposed to increase luminosity of the RHIC collider for heavy ion beams at low energies. Luminosity decreases as the square of bunch intensity due to the beam loss from the RF bucket as a result of the longitudinal intra beam scattering (IBS), as well as due to the transverse emittance growth because of the transverse IBS. Both transverse and longitudinal IBS can be counteracted with electron cooling. This would allow one to keep the initial peak luminosity close to constant throughout the store essentially without the beam loss. In addition, the phase-space density of the hadron beams can be further increased by providing stronger electron cooling. Unfortunately, the defining limitation for low energies in RHIC is expected to be the space charge.

Here we explore an idea of additional improvement in luminosity, on top of the one coming from just IBS compensation and longer stores, which may be expected if one can operate with longer bunches at the space-charge limit in a collider. This approach together with electron cooling may result in about 10-fold improvement in total luminosity for low-energy RHIC program.

\section{Luminosity limits at low energies in RHIC}

In a collider, maximum achievable luminosity is typically limited by the beam-beam effects. However, at low-energy RHIC operation with heavy ions the dominant limitation is expected from the direct space-charge effects of the beam [1]. Since luminosity achieved in RHIC is low mostly for $\gamma<10$, this region would benefit the most from future luminosity improvement. Also note, that in most recent RHIC operation at $\gamma=10.5$ we were not yet limited by the space-charge effects which allows some room for luminosity improvement.

Table 1 shows beam lifetimes measured in dedicated low-energy RHIC beam studies for various space-charge tunes shifts under collisions (column "comments" lists some specifics of different experiments such as transverse acceptance in collimators, synchrotron tune $\mathrm{Q}_{\mathrm{s}}$ and total beam-beam parameter $\xi$, see Ref. [2] for details).

\begin{tabular}{|l|l|l|ll|}
\hline$\Delta \mathrm{Q}_{\mathrm{sc}}$ & $\tau[\mathrm{s}]$ & $\gamma$ & Comments & \\
\hline 0.03 & 600 & 10 & $5 \sigma_{\mathrm{x}}, \mathrm{Q}_{\mathrm{s}}=0.002, \xi=0.002$ & (1 IP) \\
\hline 0.05 & 400 & 6.1 & $3 \sigma_{\mathrm{x}}, \mathrm{Q}_{\mathrm{s}}=0.006, \xi=0.0015$ & $(1 \mathrm{IP})$ \\
\hline 0.09 & 260 & 6.1 & $3 \sigma_{\mathrm{x}}, \mathrm{Q}_{\mathrm{s}}=0.006, \xi=0.0027$ & (1 IP) \\
\hline 0.1 & 70 & 4.1 & $2.2 \sigma_{\mathrm{x}}, \mathrm{Q}_{\mathrm{s}}=0.013, \xi=0.003$ (2 IP) \\
\hline
\end{tabular}

Table 1: Beam lifetime for low-energy gold ion beam for different space-charge tune shifts with collisions (with total beam-beam parameter $\xi$ ). 
In a collider, when maximum luminosity is limited by beam-beam effects it can be expressed through the beam-beam parameter $\xi$ (maximum tune shift for zero-amplitude particles):

$$
\xi=\frac{Z^{2} r_{p}}{A} \frac{N_{i}}{4 \pi \beta^{2} \gamma \varepsilon} \frac{1+\beta^{2}}{2}
$$

as

$$
L=\frac{A}{Z^{2} r_{p}} \frac{N_{i} c}{\beta^{*} C_{r}} \frac{2 \gamma \beta^{3}}{1+\beta^{2}} f\left(\frac{\sigma_{s}}{\beta^{*}}\right) \xi
$$

where $\beta^{*}$ is the beta function at the interaction point (IP), $r_{p}$ is the proton classical radius, $N_{\mathrm{i}}$ is the number of ions per bunch, $A$ and $Z$ are the ion atomic and charge numbers, $\gamma, \beta$ are relativistic factors, $\varepsilon$ is the un-normalized rms emittance, $\sigma_{s}$ is the rms bunch length, and $C_{r}$ is the ring circumference. The factor $f\left(\sigma_{\mathcal{S}} / \beta^{*}\right)$, which describes the "hour-glass" effect, is close to unity when the longitudinal rms beam size is much less than the value of beta function at the IP, and decreases when $\sigma_{s}$ is increased.

In the case of the beam-beam limit, considering collisions with large crossing angles helps to reduce beam-beam parameter, which depends on the crossing angle. Thus cooling of transverse emittance and reducing in turn beta-function at collision point can help to improve overall luminosity, as suggested for high-energy RHIC operation by T. Roser.

If luminosity is limited by the space-charge tune shift value $\Delta \mathrm{Q}_{\mathrm{sc}}$, it can be expressed as:

$$
L=\frac{A}{Z^{2} r_{p}} \frac{N_{i} c}{\beta^{*}} \frac{\sqrt{2 \pi} \sigma_{s}}{C_{r}{ }^{2}} \gamma^{3} \beta^{3} f\left(\frac{\sigma_{s}}{\beta^{*}}\right) \Delta Q_{s c}
$$

where no transverse acceptance limitation was taken into account. For a Gaussian transverse distribution, the maximum incoherent space-charge tune shift can be estimated as:

$$
\Delta Q_{s c}=-\frac{Z^{2} r_{p}}{A} \frac{N_{i}}{4 \pi \beta^{2} \gamma^{3} \varepsilon} \frac{C_{r}}{\sqrt{2 \pi} \sigma_{s}},
$$

where we assumed round beam transversely and Gaussian longitudinal profile.

When in addition to the space charge one also has limitation from the transverse acceptance (which is the case for RHIC at lowest energies of interest) even stronger drop in luminosity with energy should be expected, as was pointed out by V. Lebedev [3]:

$$
L=8 \pi^{2}\left(\frac{A}{Z^{2} r_{p}}\right)^{2} \frac{c \varepsilon}{\beta^{*}} \frac{\sigma_{s}^{2}}{C_{r}{ }^{3}} \gamma^{6} \beta^{5} f\left(\frac{\sigma_{s}}{\beta^{*}}\right) \Delta Q_{s c}^{2}
$$

Figure 1 shows the limits for a single-bunch luminosity in RHIC due to the space charge for several space-charge tune spreads $\Delta \mathrm{Q}_{\mathrm{sc}}=0.03,0.05,0.1$ (Eq. (3)), as well as if luminosity would be limited by beam-beam with a total tune spread of $\xi=0.015$. However, one cannot reach such large beam-beam parameter for low energies in RHIC without exceeding the space-charge limit first. 
The ratio of the space-charge to beam-beam tune spread is given by

$$
\frac{\Delta Q_{s c}}{\xi}=\frac{1}{\gamma^{2}} \frac{C_{r}}{\sqrt{2 \pi} \sigma_{s}} \frac{2}{1+\beta^{2}}
$$

and is plotted in Fig. 2 for a typical length of ion bunches in RHIC at low energies $\left(\sigma_{\mathrm{s}}=1-2 \mathrm{~m}\right)$. One can see that space charge is the dominant limiting effect for energies below $\gamma_{\mathrm{tr}}=23$, which is the region of most interest for the low-energy RHIC physics program.

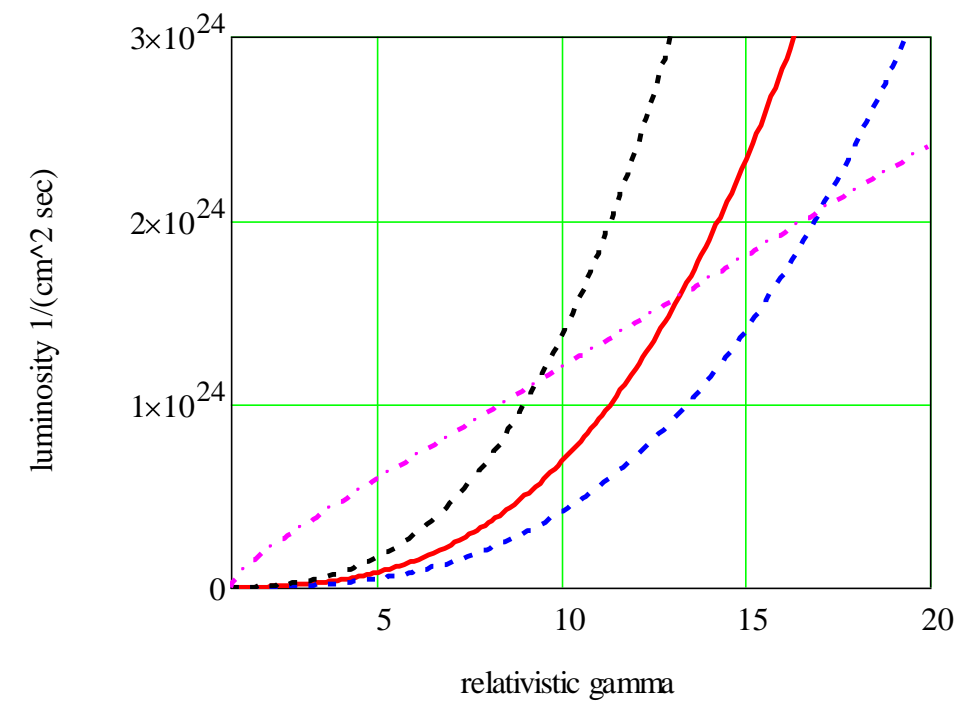

Fig. 1. Single-bunch luminosity (shown for $\beta^{*}=10 \mathrm{~m}$ ) limitation of $\mathrm{Au}$ ions in RHIC: 1) magenta line - limitation due to total beam-beam parameter of 0.015 (such values could not be reached without exceeding space-charge limit first); 2) limited by space-charge tune spread $\Delta \mathrm{Q}_{\mathrm{sc}}=0.03$ (blue, lower curve), 0.05 (red, solid middle curve) and 0.1 (black, upper dash curve).

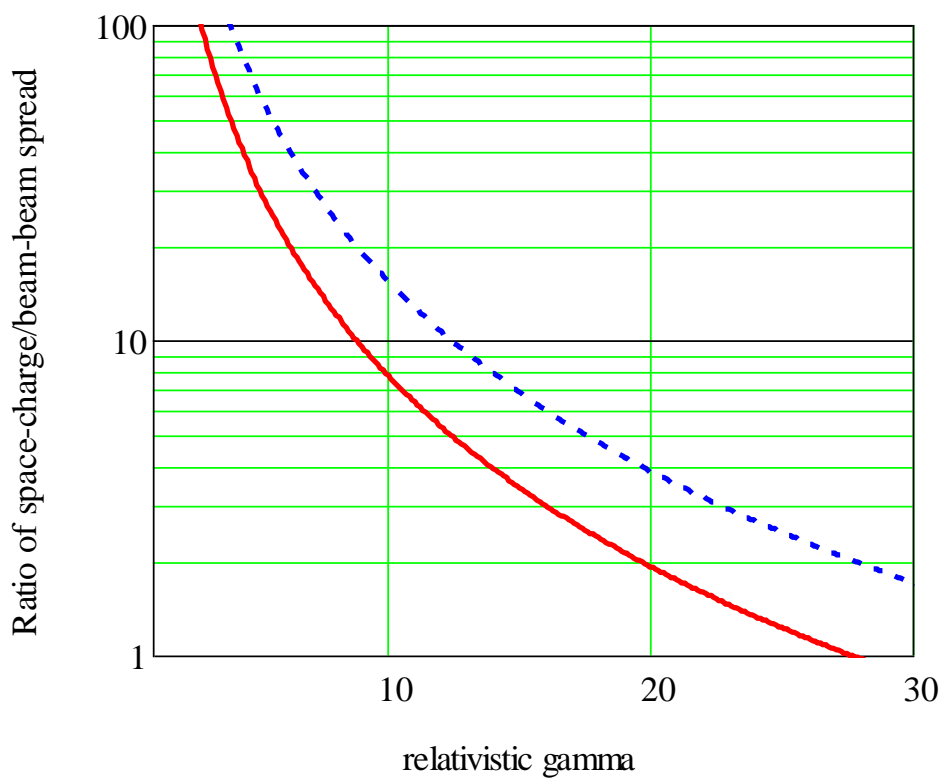

Fig. 2. Ratio of the space-charge tune spread to beam-beam spread at low energies in RHIC for rms bunch length $2 \mathrm{~m}$ (red) and $1 \mathrm{~m}$ (blue, upper dash line). 
In the case of the space-charge limit, considering collisions with large crossing angles does not help since space-charge tune shift does not depend on the crossing angle. However, one may still consider getting an additional (on top of IBS compensation) improvement from cooling.

The key to this improvement is observation that in Eq. (3) luminosity for head-on collisions does not scale with hour-glass function $f\left(\sigma_{s} / \beta^{*}\right)$ anymore but rather as $\mathrm{f}(\mathrm{x}) \cdot \mathrm{x}$, where $\mathrm{x}=\sigma_{\mathrm{s}} / \beta^{*}$. Thus, going to a longer bunch length helps to control the space-charge effect while improving luminosity simultaneously [3-4]. Figures 3 and 4 show functions $f(x)$ and $f(x) \cdot x$, respectively.

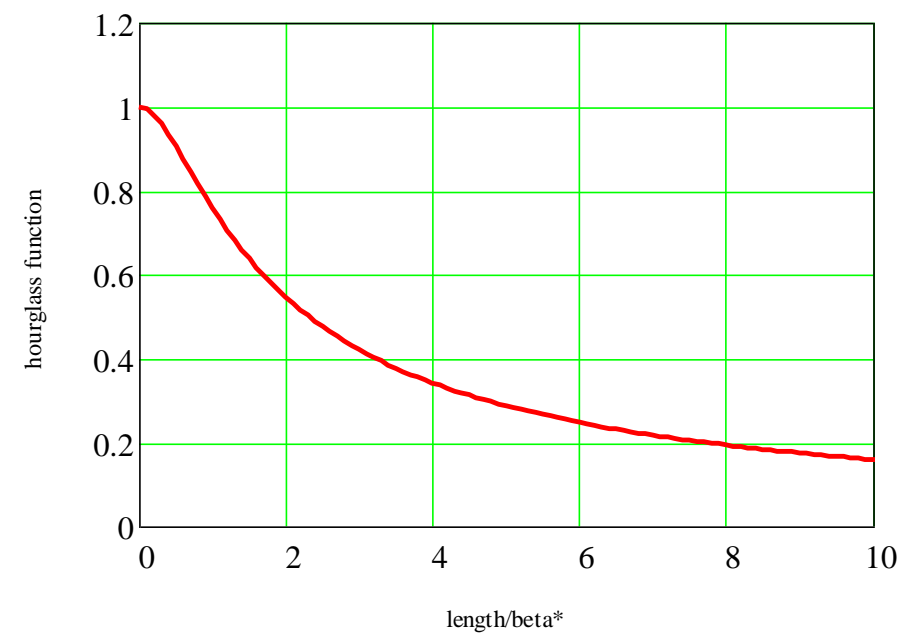

Fig. 3. Hour-glass function $f\left(\sigma_{s} / \beta^{*}\right)$ vs. $\left(\sigma_{s} / \beta^{*}\right)$.

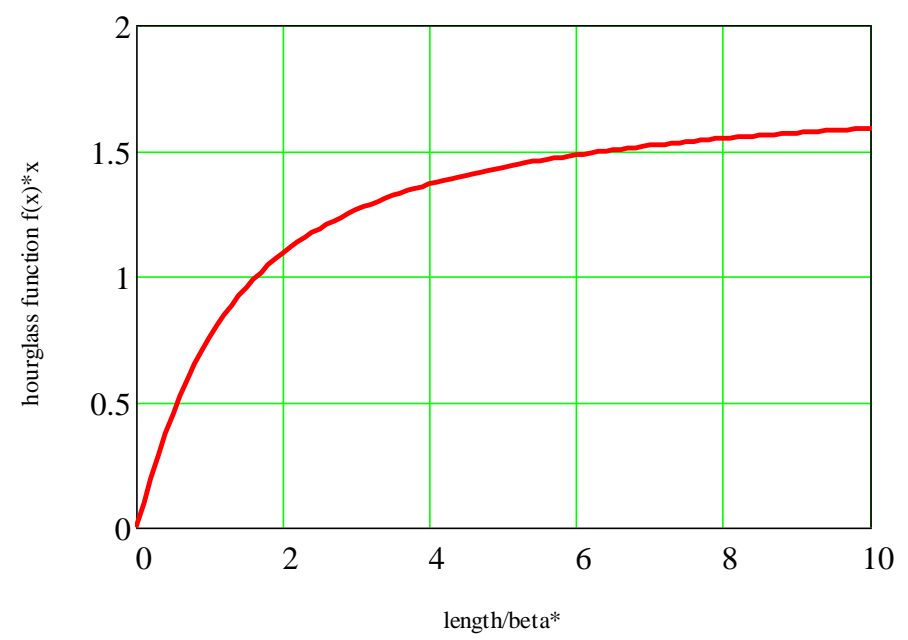

Fig. 4. Hour-glass function $\left[f\left(\sigma_{\mathcal{S}} / \beta^{*}\right) \cdot\left(\sigma_{\mathcal{S}} / \beta^{*}\right)\right]$ vs. $\left(\sigma_{\mathcal{S}} / \beta^{*}\right)$. 


\section{Luminosity with longer bunches and cooling}

As an example of additional luminosity improvement from long bunches, let's take initial rms bunch length of $\sigma_{\mathrm{s}}=1 \mathrm{~m}$ and $\beta^{*}=5 \mathrm{~m}$. If we can have longer bunch by going from $28 \mathrm{MHz}$ to $9 \mathrm{MHz}$ $\mathrm{RF}$ this will allow us to cool transverse emittance by the same amount (keeping the space-charge tune shift constant) and, in turn, it should allow us to reduce $\beta^{*}$ by the same amount, by having the same beam size in the triplets.

Assuming that bunch length can be increased by a factor of 3 to $\sigma_{\mathrm{s}}=3 \mathrm{~m}(\mathrm{rms})$, we can now cool transverse emittance by a factor of 3 (keeping the space-charge tune shift constant), which allows us to reduce $\beta^{*}$ by a factor of 3 as well (new $\beta^{*}=5 / 3 \mathrm{~m}$ ). As a result, we get:

$$
\frac{f(9 / 5) * 9 / 5}{f(1 / 5) * 1 / 5}=5.3
$$

This is an additional factor in luminosity improvement on top of the factor reported before which came mostly from compensation of IBS with cooling [5]. Such an approach of operation under the space-charge limit (head-on but with longer bunches), may allow us significant overall improvement in total luminosity with electron cooling at low energies. For example, one can achieve total luminosity $>1 \cdot 10^{27} 1 / \mathrm{cm}^{2} \mathrm{~s}^{-1}$ at $\gamma=10.7\left(\sqrt{s_{N N}}=20 \mathrm{GeV}\right)$. Note that such estimate of additional luminosity improvement refers to a "total luminosity" without the vertex cut in the detector. However, for RHIC application, the question of useful luminosity within the detector vertex cut becomes of most interest. Figure 5 shows maximum luminosity as a function of vertex cut with and without cooling for $\gamma=4.1\left(\sqrt{s_{N N}}=7.7 \mathrm{GeV}\right)$. Since without cooling luminosity cannot be kept constant due to IBS, which results in a significant beam loss, the maximum luminosity without cooling in Fig. 6 was divided by a factor of 3 to account for the luminosity lifetime and refill time needed between short stores. Similar improvement in luminosity could be expected for other energies as well, as shown in Fig. 7 for $\gamma=10.5$, for example.

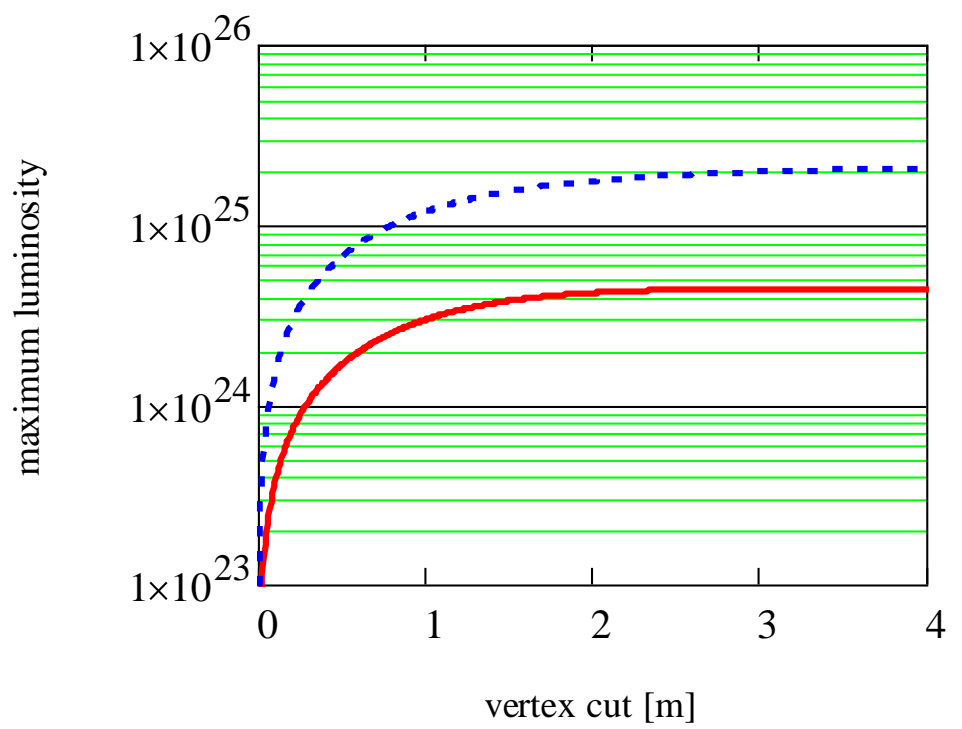

Fig. 5. Maximum luminosity for 111 bunches of $\mathrm{Au}$ ions in RHIC at $\gamma=4.1$ and space-charge tune spread $\left.\Delta \mathrm{Q}_{\mathrm{sc}}=0.05: 1\right)$ with electron cooling and long bunches $\left(\sigma_{\mathrm{s}}=4.5 \mathrm{~m}, \beta^{*}=2 \mathrm{~m}, \varepsilon_{\mathrm{n}, 95 \%}=5 \mu \mathrm{m}\right.$, $\left.\mathrm{N}_{\mathrm{i}}=5 \mathrm{e} 8\right)$ - blue, dash curve; 2) without cooling $\left(\sigma_{\mathrm{s}}=1.5 \mathrm{~m}, \beta^{*}=6 \mathrm{~m}, \varepsilon_{\mathrm{n}, 95 \%}=15 \mu \mathrm{m}, \mathrm{N}_{\mathrm{i}}=5 \mathrm{e} 8\right)-$ red, solid lower curve. 


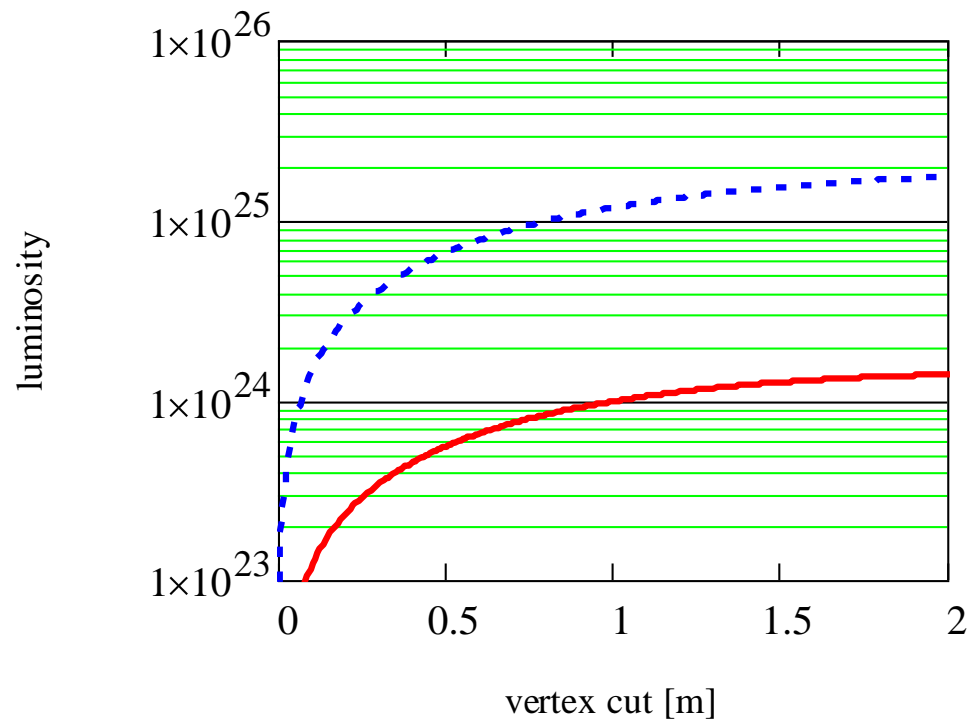

Fig. 6. Average luminosity for 111 bunches of Au ions in RHIC at $\gamma=4.1$ and space-charge tune spread $\Delta \mathrm{Q}_{\mathrm{sc}}=0.05:$ 1) with electron cooling and long bunches $\left(\sigma_{\mathrm{s}}=4.5 \mathrm{~m}, \beta^{*}=2 \mathrm{~m}, \varepsilon_{\mathrm{n}, 95 \%}=5 \mu \mathrm{m}\right.$, $\left.\mathrm{N}_{\mathrm{i}}=5 \mathrm{e} 8\right)$ - blue, dash curve; 2) without cooling $\left(\sigma_{\mathrm{s}}=1.5 \mathrm{~m}, \beta^{*}=6 \mathrm{~m}, \varepsilon_{\mathrm{n}, 95 \%}=15 \mu \mathrm{m}, \mathrm{N}_{\mathrm{i}}=5 \mathrm{e} 8\right)-$ red, solid curve. Here, maximum luminosity without cooling was divided by a factor of 3 to account for luminosity lifetime due to IBS and time between refills.

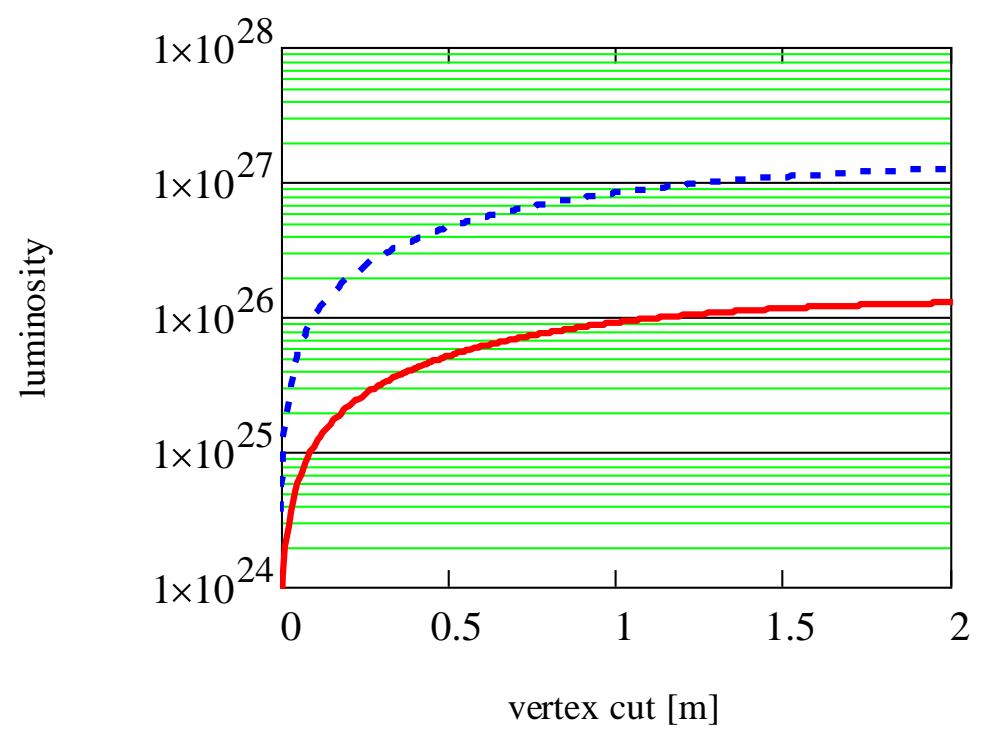

Fig. 7. Average luminosity for 111 bunches of Au ions in RHIC at $\gamma=10.5$ and space-charge tune spread $\left.\Delta \mathrm{Q}_{\mathrm{sc}}=0.05: 1\right)$ with electron cooling and long bunches $\left(\sigma_{\mathrm{s}}=3 \mathrm{~m}, \beta^{*}=1 \mathrm{~m}, \varepsilon_{\mathrm{n}, 95 \%}=5 \mu \mathrm{m}\right.$, $\left.\mathrm{N}_{\mathrm{i}}=2.1 \mathrm{e} 9\right)$ - blue, dash curve; 2) without cooling $\left(\sigma_{\mathrm{s}}=1 \mathrm{~m}, \beta^{*}=3 \mathrm{~m}, \varepsilon_{\mathrm{n}, 95 \%}=15 \mu \mathrm{m}, \mathrm{N}_{\mathrm{i}}=2.1 \mathrm{e} 9\right)$ - red, solid lower curve. Here, maximum luminosity without cooling was divided by a factor of 3 to account for luminosity lifetime due to IBS and time between refills. 
Overall expected luminosity improvement as a function of energy is shown in Fig. 8, where we assumed that with cooling maximum luminosity can be kept constant. One can see that about 10-fold improvement from cooling may be expected for the total luminosity without the vertex cut. The improvement factor is reduced when the vertex cut is decreased but it is still significant, as shown in Figs. 5-7 for selected energies.

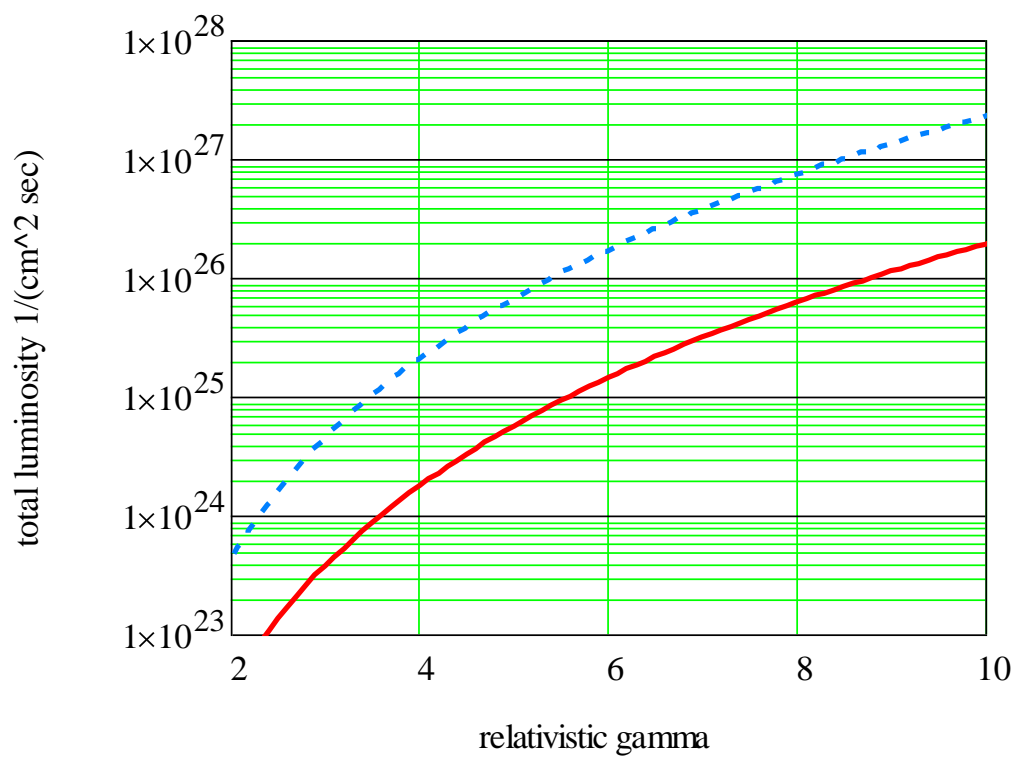

Fig. 8. Projection of total (without vertex cut) luminosity for 111 bunches of Au ions in RHIC for the space-charge tune spread of $\Delta \mathrm{Q}_{\mathrm{sc}}=0.05$ with electron cooling and long bunches (blue, dash upper curve) and without cooling (red, solid lower curve).

\section{Luminosity with longer bunches and without cooling}

We also explored an idea of improving luminosity with longer bunches but without electron cooling. In such a case, having longer bunch allows us to put higher intensity per bunch which can boost maximum luminosity dramatically. Unfortunately, without the help of cooling increased bunch intensity results in very large IBS growth rates with fast beam losses from the RF bucket and on the transverse acceptance. As a result, luminosity lifetime becomes extremely short so that such approach requires also very short stores and is getting closer to the "top-off" mode with a continuous refill which was considered before for the low-energy operation.

The luminosity lifetime in such approach was studied using the BETACOOL code [6], including losses from the RF bucket and on the transverse acceptance. As an example, we show results of such approach for $\gamma=4.1\left(\sqrt{s_{N N}}=7.7 \mathrm{GeV}\right)$.

In simulations, the rms bunch length was increased to $4.5 \mathrm{~m}$ (9 MHz RF, $40 \mathrm{kV})$, which allowed us to increase bunch intensity to $\mathrm{N}=1.5 \mathrm{e} 9$, keeping the space-charge tune shift constant. Consequently, this resulted in very large IBS growth rates which led to a fast drop of luminosity due to beam losses. As a result, the store length was shortened to just 6 min in order to get higher average luminosity per store. To take into account results of this simulation, in Fig. 9, maximum luminosity for such approach (no cooling, high intensity) was divided by a factor of 4 to represent average luminosity within a very short store. Figure 9 shows comparison of such luminosity without cooling with other cases discussed above. One can see that such approach (longer bunches, higher intensity, shorter stores) can increase average luminosity significantly even without cooling but it 
will also come with heavy beam losses which was already an issue in RHIC operation at lower energies even for nominal bunch intensities. On the contrary, the use of electron cooling should help to minimize beam losses and make operation easier with longer stores in addition to a significant luminosity improvement. Simulations of luminosity evolution with time for the three cases presented in Fig. 9 are shown in Fig. 10, for completeness.

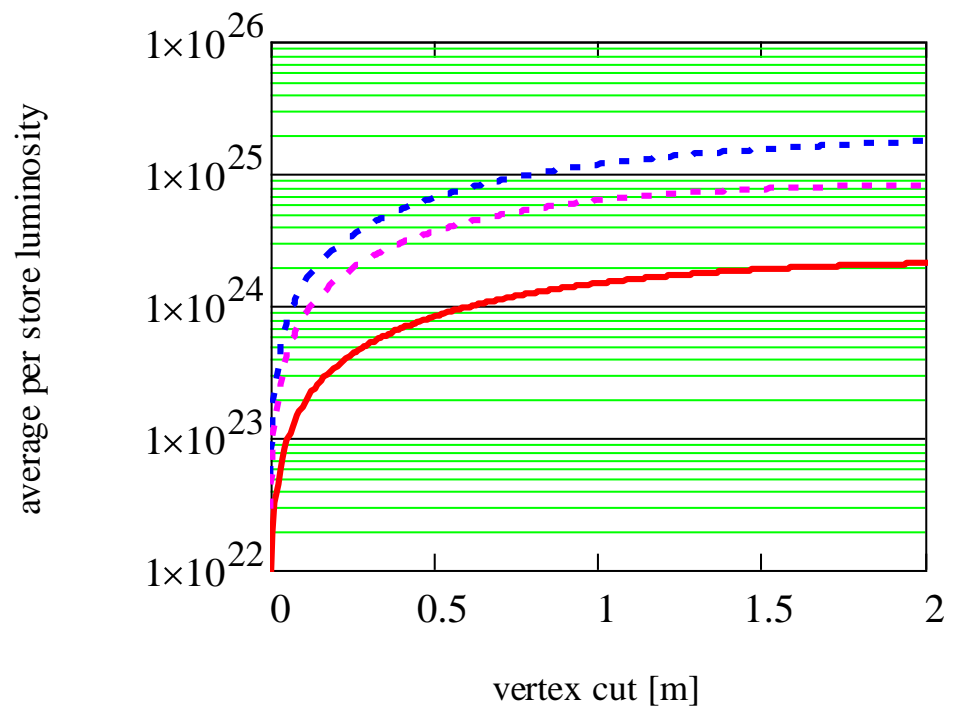

Fig. 9. Average (per store) luminosity for 111 bunches of $\mathrm{Au}$ ions in RHIC at $\gamma=4.1$ and spacecharge tune spread $\Delta \mathrm{Q}_{\mathrm{sc}}=0.05:$ 1) electron cooling and long bunches $\left(\sigma_{\mathrm{s}}=4.5 \mathrm{~m}, \beta^{*}=2 \mathrm{~m}\right.$, $\left.\varepsilon_{\mathrm{n}, 95 \%}=5 \mu \mathrm{m}, \mathrm{N}_{\mathrm{i}}=5 \mathrm{e} 8\right)$ - blue, dash curve; 2) without cooling $\left(\sigma_{\mathrm{s}}=1.5 \mathrm{~m}, \beta^{*}=6 \mathrm{~m}, \varepsilon_{\mathrm{n}, 95 \%}=15 \mu \mathrm{m}\right.$, $\mathrm{N}_{\mathrm{i}}=5 \mathrm{e} 8$ ) - red, solid curve; 3) without cooling but longer bunches with higher bunch intensity $\left(\sigma_{\mathrm{s}}=4.5 \mathrm{~m}, \beta^{*}=6 \mathrm{~m}, \varepsilon_{\mathrm{n}, 95 \%}=15 \mu \mathrm{m}, \mathrm{N}_{\mathrm{i}}=1.5 \mathrm{e} 9\right)$ - magenta, middle curve (maxi mum luminosity was divided by a factor of 4 to account for a very short luminosity lifetime - in simulations, the luminosity was decreased by a factor of 4 after first 3 min as shown in Fig. 10).

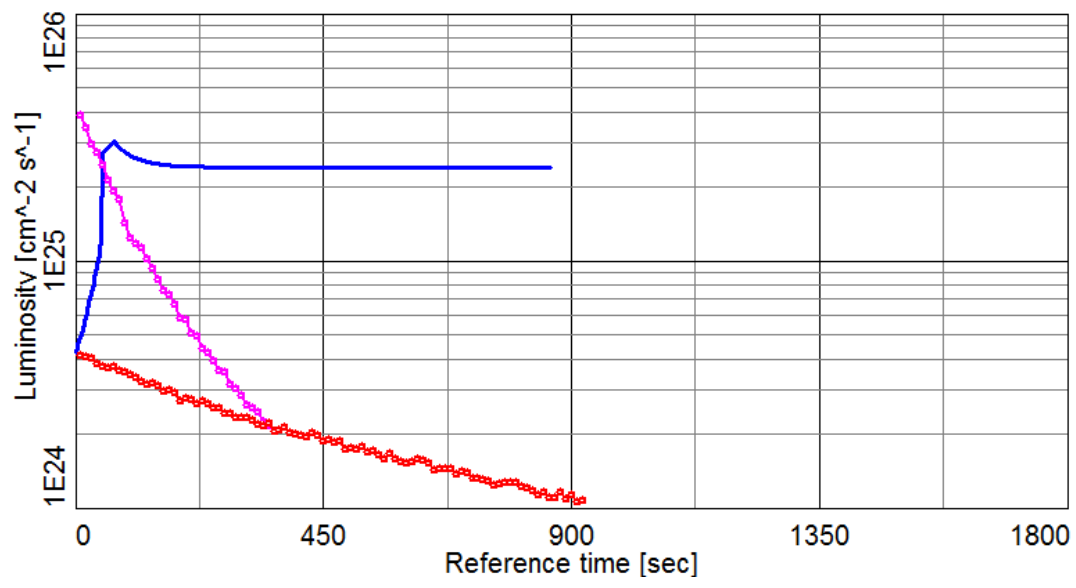

Fig. 10. Simulated luminosity evolution for 3 cases summarized in Fig. 9: 1) electron cooling and long bunches $\left(\sigma_{\mathrm{s}}=4.5 \mathrm{~m}, \beta^{*}=2 \mathrm{~m}, \varepsilon_{\mathrm{n}, 95 \%}=5 \mu \mathrm{m}, \mathrm{N}_{\mathrm{i}}=5 \mathrm{e} 8\right)-$ blue, top curve; 2) without cooling $\left.\left(\sigma_{\mathrm{s}}=1.5 \mathrm{~m}, \beta^{*}=6 \mathrm{~m}, \varepsilon_{\mathrm{n}, 95 \%}=15 \mu \mathrm{m}, \mathrm{N}_{\mathrm{i}}=5 \mathrm{e} 8\right)-\mathrm{red} ; 3\right)$ without cooling but longer bunches with higher bunch intensity $\left(\sigma_{\mathrm{s}}=4.5 \mathrm{~m}, \beta^{*}=6 \mathrm{~m}, \varepsilon_{\mathrm{n}, 95 \%}=15 \mu \mathrm{m}, \mathrm{N}_{\mathrm{i}}=1.5 \mathrm{e} 9\right)-$ magenta. 


\section{Summary}

Low-energy RHIC physics program would greatly benefit from a significant luminosity increase at low energies. It was reported before that using electron cooling to counteract IBS at these energies and providing longer physics stores would increase luminosity significantly, with the limitation imposed by the space-charge effects.

Here we showed that an additional improvement in luminosity may be possible by operating with longer bunches at the space-charge limit in a collider, which further extends benefits from cooling at low energies. Overall improvement from cooling could be about 10-fold gain in total luminosity with significant improvement of luminosity even for a short vertex cut of the detector.

We also explored a possibility of luminosity improvement by using longer bunches with higher intensity without cooling. Although gain in maximum luminosity is significant the luminosity lifetime in such approach becomes very short and requires very short stores in order to maximize gain in integrated luminosity.

\section{Acknowledgement}

We would like to thank I. Ben-Zvi, W. Fischer, V. Litvinenko and T. Roser for useful discussions on this subject.

\section{References}

[1] A. Fedotov et al., "Beam dynamics limits for low-energy RHIC operation", Proc. of HB2008 Workshop (Nashville, TN, 2008), p. 75.

[2] A. Fedotov et al., "Beam lifetime and limitations during low-energy RHIC operation", Proc. of PAC11 (New York, NY, 2011), p. 2285, THP081.

[3] V. Lebedev (presentation at NICA meeting, January 2010).

[4] A. Fedotov, ’Luminosity scaling for eRHIC under space-charge limit" (2010).

[5] A. Fedotov et al., Proc. of PAC11 (New York, NY, 2011), p. 2288, THP082.

[6] BETACOOL code, http://betacool.jinr.ru. 